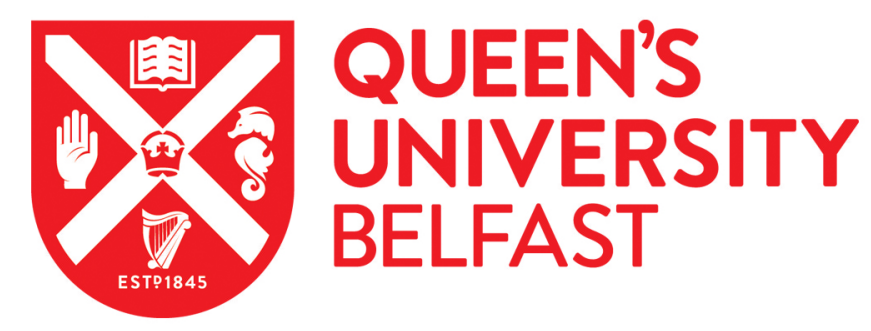

\title{
Decomposing the educational gradient in allostatic load across European populations. What matters the most: differentials in exposure or in susceptibility?
}

Veronesi, G., Kee, F., Hicks, B., Forrest, H., Tunstall-Pedoe, H., Kuulasmaa, K., Sans, S., Salomaa, V., Thorand, B., Di Castelnuovo, A., Soderberg, S., Cesana, G., Bobak, M., De Ponti, R., lacoviello, L., Palmieri, L., Zeller, T., Blankenberg, S., \& Ferrario, M. M. (2020). Decomposing the educational gradient in allostatic load across European populations. What matters the most: differentials in exposure or in susceptibility? Journal of Epidemiology \& Community Health, 74(12), 1008-1015. https://doi.org/10.1136/jech-2020-213946

Published in:

Journal of Epidemiology \& Community Health

Document Version:

Peer reviewed version

Queen's University Belfast - Research Portal:

Link to publication record in Queen's University Belfast Research Portal

\section{Publisher rights}

Copyright 2020 the authors.

This is an open access Creative Commons Attribution-NonCommercial License (https://creativecommons.org/licenses/by-nc/4.0/), which permits use, distribution and reproduction for non-commercial purposes, provided the author and source are cited.

\section{General rights}

Copyright for the publications made accessible via the Queen's University Belfast Research Portal is retained by the author(s) and / or other copyright owners and it is a condition of accessing these publications that users recognise and abide by the legal requirements associated with these rights.

\section{Take down policy}

The Research Portal is Queen's institutional repository that provides access to Queen's research output. Every effort has been made to ensure that content in the Research Portal does not infringe any person's rights, or applicable UK laws. If you discover content in the

Research Portal that you believe breaches copyright or violates any law, please contact openaccess@qub.ac.uk. 


\section{Decomposing the educational gradient in allostatic load across European populations. What matters the most: differentials in exposure or in susceptibility?}

Veronesi $\mathrm{G}^{1}$, Kee $\mathrm{F}^{2}$, Hicks $\mathrm{B}^{2}$, Forrest $\mathrm{H}^{1}$, Tunstall-Pedoe $\mathrm{H}^{3}$, Kuulasmaa $\mathrm{K}^{4}$, Sans $\mathrm{S}^{5}$, Salomaa $\mathrm{V}^{4}$, Thorand $\mathrm{B}^{6}$, Di Castelnuovo $\mathrm{A}^{7}$, Soderberg $\mathrm{S}^{8}$, Cesana $\mathrm{G}^{9}$, Bobak $\mathrm{M}^{10}$, De Ponti $\mathrm{R}^{11}$, Iacoviello $\mathrm{L}^{1,12}$, Palmieri $\mathrm{L}^{13}$, Zeller $\mathrm{T}^{14,15}$, Blankenberg $\mathrm{S}^{14,15}$, Ferrario $\mathrm{MM}^{1}$ on behalf of the BiomarCaRE Consortium

1. Research Center in Epidemiology and Preventive Medicine, Department of Medicine and Surgery, University of Insubria, Varese, Italy

2. Centre for Public Health, Queens University of Belfast, Belfast, United Kingdom

3. Cardiovascular Epidemiology, Institute of Cardiovascular Research, University of Dundee, Scotland, UK

4. Dept. of Public Health Solutions, Finnish Institute for Health and Welfare, Helsinki, Finland.

5. Catalan Department of Health, Barcelona, Spain

6. Institute of Epidemiology, Helmholtz Zentrum München, German Research Center for Environmental Health, Neuherberg, Germany

7. Mediterranea Cardiocentro, Napoli, Italy

8. Department of Public Health and Clinical Medicine, Umeå University, Umeå, Sweden.

9. Research Centre on Public Health, Department of Medicine and Surgery, University of Milano Bicocca, Monza, Italy

10. Department of Epidemiology and Public Health, University College London, London, UK

11. Department of Medicine and Surgery, University of Insubria, Varese, Italy

12. Department of Epidemiology and Prevention, IRCCS Neuromed, Pozzilli, Italy

13. Department of Cardiovascular, Endocrine-metabolic Diseases, and Ageing, National Institute of Health-ISS, Rome, Italy

14. University Heart \& Vascular Center Hamburg, Department of Cardiology, Hamburg, Germany

15. German Center for Cardiovascular Research, Partner Site Hamburg/Kiel/Lübeck, Hamburg, Germany

\section{Corresponding Author}

Prof. Marco M Ferrario

Research Center in Epidemiology and Preventive Medicine

Department of Medicine and Surgery

University of Insubria

Via O. Rossi 9

21100 Varese - Italy

marco.ferrario@uninsubria.it

Phone: +390332 217225

Word count: 3553 


\section{Abstract (word count: 243)}

Background: We investigate whether socially disadvantaged individuals are more susceptible to the detrimental effects of smoking and alcohol intake on allostatic load (AL), a marker of physiological "wear and tear", resulting from adaptation to chronic stress.

Methods: In a cross-sectional analysis, 27019 men and 26738 women aged 35-74 were identified from 21 European cohorts in the BiomarCaRE consortium. We defined three educational classes (EDs) according to years of schooling and an AL score as the sum of zscores of 8 selected biomarkers from the cardiovascular, metabolic, and inflammatory systems. We used the Oaxaca-Blinder decomposition to disentangle the educational class gradient in AL score into the differential exposure (DE, attributable to different distribution of smoking and alcohol intake across EDs) and the differential susceptibility (DS, attributable to a different effect of risk factors on AL across EDs) components.

Results: Less educated men (mean AL difference: 0.68, 95\%CI: 0.57-0.79) and women $(1.52,1.40-1.64)$ had higher AL scores. DE accounted for $7 \%$ and $6 \%$ of the gradient in men and women, respectively. In men, combining smoking and alcohol intake, DS accounted for $42 \%$ of the gradient, (smoking DS coefficient $=0.177,26 \%$ of the gradient; alcohol DS coefficient $=0.109 ; 16 \%$, not statistically significant). DS contribution increased to $69 \%$ in metabolic markers. DS estimates were consistent across age groups; irrespective of comorbidities; and robust to unmeasured confounding. No DS was observed in women. Conclusions: In men, a differential susceptibility mechanism substantially contributes to the educational class gradient in allostatic load.

Key words: allostatic load; educational class; differential susceptibility; Oaxaca-Blinder decomposition; cardiovascular risk; social inequalities 


\section{What is already known on this subject?}

Differential exposure to behavioral and biological risk factors accounts for a limited proportion of social inequalities in cardiovascular diseases

Literature hypothesized that less educated individuals may be more susceptible than more educated ones to the detrimental effects of risk factors, the causal pathways for such differential susceptibility mechanism remaining unclear so far

We used a novel method to disentangle the educational gradient in allostatic load, a marker of cumulative biological burden and early ageing, into differential exposure and differential susceptibility to smoking and alcohol intake.

\section{What this study adds?}

Less educated men and women had higher allostatic load score as compared to their more educated counterparts. The differential exposure mechanism accounted for $6-7 \%$ of this gradient in both gender groups.

In men, but not in women, $42 \%$ of the gradient was due to the putative differential susceptibility mechanism. Individuals with optimal risk factor profiles and no clinical manifestations of adverse health outcomes were amongst the most "frail" subgroups

In men, a differential susceptibility mechanism substantially contributes to the educational class gradient in allostatic load. 


\section{Introduction}

In European populations, low education accounts for 343 and 170 additional incident coronary heart disease events per 100000 person-years in men and women, respectively [1]. In many of the same populations, low education is also associated with higher stroke incidence, though with some geographic heterogeneity, especially in women [2]. The extent to which clinical, biological and behavioural risk factors play a role in explaining these associations is less known. Results from observational and Mendelian randomization studies converge in attributing to differential exposure (DE) to risk factors, up to half of the educational class inequalities in disease rates [1-3]. In addition to DE, recent literature hypothesizes that the effects of risk factors on cardiovascular disease onset may differ across social strata, a mechanism named "differential susceptibility" (DS) [4]. A few studies have estimated the role of DS in social disparities in cardiovascular diseases, adopting different methodological approaches including additive interaction [5-6], mediation [7], and the Oaxaca-Blinder decomposition [8]. Two of them found a disproportionate effect of smoking in low educational classes, supporting the DS hypothesis [5-6].

These reports [5-8] could not elucidate the underlying pathways for the interplay between social class and the effects of risk factors that is attributable to differential susceptibility. One candidate mechanism is the accumulation, from a very young age, of psychosocial stressors and allostatic load (AL) among the socially disadvantaged [4, 9]. Allostatic load refers to the "wear and tear" on the body resulting from mal-adaptation to chronic stressors $[10,11]$. According to causal conjectures in the literature, the association between social class and AL may be mediated by several psychosocial and behavioural risk factors; and AL itself may then be a mediator of the relationship between socio-economic status and cardiovascular diseases $[12,13]$. Thus, the differential susceptibility mechanism may be present at any step of this complex theory-informed [14] pathway, determining a disproportionate accumulation 
of allostatic load among socially disadvantaged individuals, and potentially a disproportionate effect of AL on disease outcomes, respectively. A number of previous studies have investigated the social class gradient in AL [12], although none of them ascertained the presence of differential susceptibility. Therefore, in this paper, we disentangle the contribution of smoking and alcohol intake to the educational class gradient in allostatic load in terms of differential exposure and susceptibility in a cross-sectional setting using baseline data of 21 population-based cohorts across nine European countries. The mediating role of allostatic load on the association between education and cardiovascular disease risk will be the topic of a separate, companion paper based on the same cohorts.

\section{Methods}

\section{Study overview}

The current study is based on population-based cohorts included in the BiomarCaRE (Biomarker for Cardiovascular Risk Assessment in Europe; www.biomarcare.eu) consortium [15] and in the MORGAM (MONICA Risk Genetics Archiving and Monograph) project [16] with harmonized data on education, lifestyles and the biological and clinical markers required for the derivation of the allostatic load score $(n=21)$. Study populations included men and women aged 35 to 74, except for Belfast (men only, aged 49 to 60), Brianza and Catalonia where the upper age limit was 66 and 67, respectively. Study cohorts are quite homogeneous with respect to the ethnic group, as nearly all participants are from European ethnic origin. Full details of the included cohorts are documented at https://www.thl.fi/publications/morgam/cohorts/index.html; major study characteristics, including recruitment periods and participation rates, are summarized in the Supplementary Material (Table S1). All participating studies adhered to the Declaration of Helsinki and received approval by local ethics review boards. 


\section{Definition of educational classes}

At baseline, each cohort collected information on the number of years of schooling, with high question comparability across populations and low prevalence of missing data [17]. We derived three categories of education (high, intermediate and low) from population-, sex- and birth cohort-specific tertiles of the distribution of years of schooling [18]. Mean years of schooling in the three classes were 15,10 and 8 , respectively. This classification mitigates the effect on educational classes' distribution due to differences in schooling systems across countries and time periods [18].

\section{Allostatic Load score}

To measure allostatic load, we selected eight markers among those commonly used in literature [11-12] and representing the cardiovascular (systolic and diastolic blood pressure), the metabolic (Body Mass Index, total- and HDL-cholesterol, triglycerides, blood glucose), and the inflammatory (C-Reactive Protein) systems. In one population (KORA-Augsburg), glycated haemoglobin replaced blood glucose. Measurement details for the markers included into the AL score are in the in the Supplementary Material (Table S2). For each marker, we derived the Z-score, i.e. positive or negative standard deviations from the population-, sexand fasting status-specific mean. Before standardization, markers with a positively skewed distribution were log-transformed. We defined an AL score as the sum of the $8 \mathrm{Z}$-scores [11, 19]; the distribution of the score by population and sex is reported as Supplementary Material (Figure S1). Similarly, we also obtained 3 AL sub-scores corresponding to the secondary outcomes (cardiovascular, metabolic and inflammatory) by summing-up the Z-scores for the relevant markers only.

Assessment of lifestyles and anamnestic risk factors 
Cigarette smoking and alcohol intake were ascertained by interview or self-reported questionnaire. We considered a 5-class variable defined as never smokers, former smokers, and 3 categories of current smokers according to the number of cigarettes/day smoked $(\leq 10$, $11-20,>20$ ). Daily alcohol intake (in grams) was converted to average drinks per day, considering 12.5 grams of alcohol as a standard drink [20]. We further categorised alcohol intake as abstainers ( 0 grams of alcohol per day), $1-2,3-4,5$ or more drinks per day. History of cardiovascular disease (CVD: myocardial infarction, unstable angina pectoris or stroke) and diabetes at recruitment was obtained from clinical records or from questionnaires. Elevated blood pressure was defined as $\geq 140 / 90 \mathrm{mmHg}$ or self-reported use of antihypertensive treatment. We defined a binary variable as the presence, at the baseline survey, of any of the following health outcomes: elevated blood pressure, obesity (BMI $>30 \mathrm{~kg} / \mathrm{m}^{2}$ ), self-reported diabetes or history of CVD.

\section{Statistical analyses}

In total the 21 study cohorts comprised $n=62171$ men and women aged 35 to 74 at recruitment with valid data on education (Table S1 in the Supplementary Material). Of these, we excluded 5726 individuals because of missing data on one or more allostatic load markers; 1842 for missing data on lifestyles (number of cigarettes smoked or alcohol intake); 846 for missing information on cardiovascular disease, diabetes or elevated blood pressure. We decided not to pursue multiple imputation techniques, as allostatic load and the other risk factors constitute our major outcome or exposures of interest. The available sample size was 53757 (27019 men, 26738 women), corresponding to the $86.5 \%$ of the original sample, with no substantial difference in this prevalence across educational classes (Table S1).

We estimated the mean AL score by educational class and sex, and refer to an "educational class gradient in AL score" $[\Delta \mathrm{AL}]$ as the difference in the average AL score between two classes. We adopted the detailed Oaxaca-Blinder decomposition method [21], including 
cigarette smoking and alcohol intake as risk factors, and age (in classes: $35-44,45-54$ and 5574 years old) and study center as potential confounders. This method disentangles $\Delta \mathrm{AL}$ assuming an additive model with three components, one interpretable in terms of differential exposure, the other in terms of differential susceptibility, plus a residual term. We present comprehensive results contrasting low and high educational groups, and report the decomposition for low vs. intermediate education as Supplementary Material. From the interpretation viewpoint, given a positive $\Delta \mathrm{AL}$, a positive $\mathrm{DE}$ coefficient is the expected reduction in $\triangle \mathrm{AL}$ if those with a low education had, on average, the same level of risk factors as those with a high education. A positive DS coefficient is the expected reduction in $\triangle \mathrm{AL}$ if the effect of a risk factor on AL in those with a low level of education was the same as in more highly educated individuals. Full details are in the Supplementary Material. We applied the decomposition to the overall AL score, as well as to the 3 sub-scores. In addition, we also report results of stratified analyses by age groups and by the presence of any co-morbidity as defined above. In sensitivity analyses, we assess robustness of our DE and DS estimates to unmeasured confounding of the mediator-outcome relationship. Details are in the Supplementary Material. The statistical analyses were performed with the SAS software release 9.4, while the Oaxaca-Blinder decomposition was estimated using the oaxaca command in STATA [22].

\section{Results}

Less educated men and women had a higher AL score than their more educated counterparts (Table 1; both $\mathrm{p}$-values $<0.0001$ ). In both men and women, low education was associated with a higher prevalence of smoking overall and of heavy smokers, and with a lower prevalence of moderate alcohol intake (1-2 drinks/day; all p-values $<0.0001$ ). Finally, $58 \%$ of men and $53 \%$ of women had at least one comorbidity, the prevalence increasing for decreasing education in men and women (both p-values: <0.0001). After adjustment for age, 
educational class and study center, cigarette smoking and drinks/day of alcohol intake were associated with increased allostatic load score in men and women (Table S3). In women, teetotallers had an increased AL score as compared to moderate drinkers.

\section{Detailed Oaxaca-Blinder decomposition}

The contribution of smoking and alcohol to the educational gradient in AL score as estimated from the Oaxaca-Blinder decomposition, is reported in Table $2 \mathrm{a}$ and $2 \mathrm{~b}$, for men and women, respectively. In men, in aggregate, the DE and DS components corresponded to $7 \%(0.048$; 95\%CI: $0.020-0.077)$ and $42 \%(0.286 ; 0.119-0.453)$ of the observed $\Delta \mathrm{AL}$, respectively. Smoking (5.2\%) was the largest contributor to the DE component. We found a positive DS coefficient among the never-smokers $(0.147 ; 0.063-0.230)$ and for $1-2$ drinks/day of alcohol intake (0.094, not statistically significant), indicating that in these subgroups less educated men have a disproportionate allostatic load accumulation as compared to their more educated counterparts. The DS coefficient diminished and reversed its sign with increasing number of cigarettes smoked or drinks/day of alcohol intake, indicating that the more educated individuals lost their advantage with exposure to risk factors.

In women (Table $2 \mathrm{~b}$ ), the observed $\Delta \mathrm{AL}$ was 1.52 (95\%CI: $1.40-1.64)$. On aggregate, the $\mathrm{DE}$ mechanism corresponded to $6.1 \%$ of the AL gradient (0.092; 0.063-0.122), with significant contributions from both alcohol intake (4.4\%) and smoking (1.7\%). The DS coefficient was modest and not statistically significant. Of note, $79 \%$ of the observed gradient remained unexplained by the considered behavioural factors.

\section{Analysis of AL sub-scores}

In men (Table 3), we observed a positive educational gradient for all the AL sub-scores corresponding to the cardiovascular, metabolic and inflammatory systems, the largest difference being for the metabolic system AL $(0.37 ; 0.29-0.44)$. About $69 \%$ of this gradient was due to the DS component $(0.25 ; 95 \% \mathrm{CI}$ : $0.14-0.37)$, and in particular to smoking $(42 \%)$ 
and alcohol intake (27\%; Table S4). Conversely, the DE contribution was negligible. Among women (Table 3), the observed $\triangle \mathrm{AL}$ was attributable to metabolic (0.98; 0.90-1.06), cardiovascular $(0.32 ; 0.27-0.37)$ and inflammatory $(0.23 ; 0.20-0.25)$ systems. The DE mechanism contributed $8-9 \%$ of the observed gradient in the inflammation and metabolic subscores only, while the DS contribution was marginal and not statistically significant. Finally, when contrasting low with intermediate education, the gradient in allostatic load was largely attenuated, remaining statistically significant in women only (Table S5).

Stratification by age and by health status

Table 4 reports the Oaxaca-Blinder decomposition for $\Delta \mathrm{AL}$, by age group and according to the presence of any comorbidity; detailed results by condition (obesity, elevated blood pressure, diabetes and cardiovascular disease) are reported as supplementary material (Table S6).

The educational gradient in AL was significant at all ages, the largest value being observed in the youngest. With age, the contribution of DE declined, until not being statistically significant in the 55-74 years old group. Conversely, the DS component increased with age, being as high as $77 \%$ in the $45-54$ years old. In men with no co-morbidities, smoking and alcohol intake contribute to the educational gradient in allostatic load both in terms of DE and DS, corresponding to $17 \%$ and $41 \%$ of the gradient, respectively. We found a positive $\Delta \mathrm{AL}$ also among subjects with co-morbidities $(0.41 ; 95 \% \mathrm{CI}$ : $0.28-0.54)$; in these men, the DS component corresponded to $55 \%$ of the gradient.

We found a positive $\Delta \mathrm{AL}$ in women in all age groups, the largest value being observed in the youngest. DE contributed to 5\%-10\%, while again no DS coefficient reached statistical significance. We also found a positive $\Delta \mathrm{AL}$ in women without $(1.11 ; 95 \% \mathrm{CI}$ : $0.98-1.24)$ and with $(0.82 ; 0.68-0.96)$ at least one comorbidity, with a contribution of DE around $9-10 \%$. No DS coefficient was statistically significant. 


\section{Sensitivity analyses for unmeasured confounding}

Sensitivity analyses suggest that our estimates for the DE component are sensitive to unmeasured confounding, with strength and direction of bias depending on the correlations of the un-measured confounder with smoking/alcohol and with allostatic load (Supplementary Material Figure S2). Conversely, the DS component is less sensitive to unmeasured confounding: confounders with the same characteristics as neighbourhood socio-economic deprivation [23] and age would have biased our estimates by about 1\% (Figure S3).

\section{Discussion}

In middle-aged Europeans, the presence of an inverse association between education and AL score has been described in cross-sectional studies in Switzerland [24], Poland [25], Denmark [26] and in the UK [27]. These studies have limitations that may undermine the generalizability of their findings, including the lack of markers from the immune system [24], the lack of women in the sample [25], and potential selection bias due to low participation rates or high prevalence of missing data on markers for the AL score [26-27]. The current cross-sectional analysis involving 53757 individuals from 21 population-based cohorts recruited in 9 European countries, found evidence of higher mean AL scores in less educated individuals as compared to their more educated counterparts. The educational gradient was consistent in all the physiological systems included in the AL conceptual framework. The gradient was larger in women than in men, and in the youngest, as reported also by other studies $[27,28]$. In a previous study, we showed that lower levels of education accelerate the cumulative risk of incident cardiovascular disease by about 5 years from middle adulthood onwards [5]. Since AL is a marker of cumulative biological burden and early ageing [10, 11], the present analysis elucidates a plausible pathway linking low educational class to premature cardiovascular disease outcomes. While the educational gradient in AL may reduce with age $[27,28]$, the gap in cardiovascular events incidence does not [5], suggesting that the effects 
on cardiovascular risk of an early-life AL accumulation in lower social strata may not be reversible.

A plausible theoretical framework posits smoking and alcohol on the causal pathway linking low social class to high allostatic load $[12,13]$. A recent review of empirical studies provided a somewhat discordant picture, reporting some null results for the association between smoking and AL, and divergent directions for the association between alcohol intake and AL [29]. Methodological differences across studies, including early life vs. adult populations, and cross-sectional vs. longitudinal study designs, should be acknowledged. In a longitudinal assessment of 35 year old individuals, Robertson et al. found a significant mediator role for smoking, but not for alcohol, affecting the social gradient in allostatic load [13]. In our study, any alcohol intake was negatively associated with the metabolic allostatic load sub-score, which includes HDL and total-cholesterol and blood glucose. Less educated men were likely to drink more than their better educated counterparts (Table 1), resulting in a negative differential exposure coefficient and a positive differential susceptibility coefficient (Table S4). This opposite sign between two distinct mediation mechanisms may contribute to the reported heterogeneity of findings in different contexts, and warrants future investigations in longitudinal studies.

No previous study [12, 24-27] has disentangled the contribution of behavioural and other determinants of the educational class gradient in AL in terms of differential exposure and differential susceptibility. Using the Oaxaca-Blinder decomposition method, we estimated that differences in the distribution of smoking and alcohol intake accounted for $6-7 \%$ of $\Delta \mathrm{AL}$, in both men and women. In addition, we found that about $42 \%$ of $\Delta \mathrm{AL}$ in men is attributable to the differential susceptibility component, a finding consistent across age groups and presence of comorbidities. Conversely, we found no evidence of DS in women, although to some extent the pattern for smoking was similar to that observed in men. The association 
between behavioural factors and education is more heterogeneous in women than in men, depending on age groups [30] and shows a clear North/South Europe geographical pattern [2, 31]. In further post-hoc exploratory-only analyses, by restricting the sample to Southern European studies from Italy and Spain, where the educational class gradient in smoking and alcohol intake in women was homogeneous across populations and age groups, we found a statistically significant contribution of DS corresponding to $29 \%$ of the observed $\triangle \mathrm{AL}$ (data not shown). Our estimated DS coefficients for smoking imply that being a never smoker is less protective in terms of AL accumulation in the less educated as compared to more educated men, this disadvantage reducing with exposure to heavy smoke.

This finding is consistent with the study by Fiorito et al., who found an educational gradient in DNA methylation (an epigenetic score of age acceleration) among non-smokers [32]. Since part of the educational gradient in AL has genetic bases [24, 33], our results could be suggestive of a gene*environment interaction, with the exposure to behavioural factors weakening the genetic or other biological advantage. Finally, the most salient pathway through which accumulation occurs under a DS mechanism is the metabolic one, including glucose, lipids and body weight. The social gradient in AL depends on a number of nonbehavioural factors including neighbourhood deprivation [23], social disadvantage in earlylife [34], material disadvantage [13] and workplace stress and occupational toxic exposures [35]. To mitigate the disproportionate AL accumulation among people in the lower educational groups, a joint action is required to intervene both upstream on the psychosocial factors leading to AL accumulation in lower social strata [13, 23, 34-35] and downstream with policies on healthy diet and physical activity specifically targeting lower social classes, in a collaborative effort across different sectors [36].

We acknowledge some limitations and strengths of the present study. First, we do not have markers from the HPA axis and the immune system in our AL score, as these were not 
consistently measured across populations. This is a common problem in many large epidemiological studies in the field [12]. Second, a large part of the educational gradient in AL remains to be explained, especially in women. Although we considered only smoking and alcohol intake, other studies of behavioural and psychosocial factors like physical activity [13], hostility and poor sleep quality [37], or fruit/vegetable intake [24] have suggested they mediated only a small fraction $(2 \%-4 \%)$ of the socio-economic position gradient in AL. Future studies may disentangle their contribution in terms of differential exposure and susceptibility, as we did in the current analyses for a limited number of behavioural factors. Part of the residual variation in AL and its gradient may be due to measurement error, such as inaccuracies in self-reported alcohol consumption, or to selection bias, as heavy alcohol consumers are less likely to participate to population-based studies [38]. However, it is worth noting that participation rates were below $60 \%$ only in two populations, and ranged between $67 \%$ and $75 \%$ in the remaining ones. In addition, the distribution of missing data did not change across educational classes, as documented in Supplementary Material Table S1. Due to the cross-sectional nature of our study, we cannot rule out reverse causation if early life stress determining allostatic load accumulation affected the educational gradient in smoking/alcohol intake. However, allostatic load at age 9 was not associated with smoking nor drug/alcohol at age 17 in a longitudinal study on rural children in the US; while smoking at age 17 significantly mediated the association between early life stress at age 9 and allostatic load at age 17 [39]. Therefore, we consider reverse causation pathways to be an implausible explanation of our findings. Finally, we acknowledge the possibility of residual confounding on the mediator-outcome association due to external stressors other than socioeconomic position. However the empirical literature on psychosocial factors and perceived stress is not consistent $[13,24,35,38]$ and may offer little support to the notion. Our sensitivity analyses suggest that estimates of the DE component may be sensitive to 
unmeasured confounding due to factors like neighborhood deprivation index [23].

Replication of our decomposition results in other contexts and settings, maybe within a

longitudinal study, is advisable to strengthen the confidence towards a causal interpretation of our findings.

Study strengths include the use of novel techniques to estimate the presence of two putative mechanisms for social inequalities, namely DE and DS, on a very large sample size from 21 population-based European cohorts. The contemporary presence of follow-up data on the same individuals will allow us to investigate the entire hypothesized framework linking behavioural factors, allostatic load and coronary heart disease incidence in terms of differential exposure and susceptibility, in a future paper.

In conclusion, we found evidence for a disproportionate accumulation of allostatic load in men with less education, however this was not consistently observed in women. The most susceptible people were individuals with a better cardiovascular disease risk factor profile, including never smokers. In these individuals, a differential susceptibility mechanism substantially contributes to the educational class gradient in allostatic load.

\section{Funding}

The BiomarCaRE Project is funded by the EU Seventh Framework Programme (FP7/20072013) under grant agreement HEALTH-F2-2011-278913. The activities of the MORGAM Data Centre have also been sustained by recent funding from EU FP7 project CHANCES (HEALTH-F3-2010-242244). A part of the biomarker determinations in the population cohorts was funded by the Medical Research Council London (G0601463, identification No. 80983: Biomarkers in the MORGAM Populations).

Ethical approval: All participating studies adhered to the Declaration of Helsinki and are responsible for ethical approval and patient consent, according to local rules at the time of study enrolment. For different study populations, the list of approvals is the following: (study name, Ethics Committee name, approval ID):

- Northern Sweden, Research ethic Committee of Umea University, 2012-280-32M; 
- FINRISK 1997, Ethics Committee at National Public Health Institute of Finland, 38/96;

- PRIME-Northern Ireland, Office for Research Ethics Committees Northern Ireland, 06/NIR02/107;

- MONICA/KORA Augsburg, Ethik-Kommision Bayerische Landesarztekammer, 05004;

- MONICA-Brianza, Comitato Etico Azienda Ospedaliera San Gerardo - Monza, 192/2005;

- MATISS Study (Latina), Comitato Etico Istituto Superiore di Sanità, PRE/96/06;

- Moli-Sani study, Comitato Etico Università Cattolica del Sacro Cuore - Roma, Prot.Pdc.P99 (A. 931/03-138-04)/CE/2004.

- MONICA-Catalonia: Director/Board of the Institute of Health Studies, ID not assigned.

- Scottish Heart Health Extended Study (SHHEC): Tayside Health Board Dundee District, $\mathrm{DM} / \mathrm{CL} / 207$

Authors' contribution: GV, FK and MMF conceived the research question and drafted the manuscript together with $\mathrm{BH}, \mathrm{HF}$. GV conducted the statistical analyses. KK, TZ and SB are guarantor of the MORGAM/BiomarCaRE database. TPH, SaS, VS, TB, FK, MMF, SoS, CG, IL, PL are PIs of the cohorts included in the current analyses. TPH, SaS, VS, TB, DiCA, SoS, CG, IL, PL, BM, DPR, KK, TZ and SB actively contributed to the interpretation of the results and made critical revision of the manuscript drafts. All Authors read and approved the final version of the manuscript.

Data sharing: No additional data available

Competing Interest: None declared.

Licence for Publication:The Corresponding Author has the right to grant on behalf of all authors and does grant on behalf of all authors, an exclusive licence (or non exclusive for government employees) on a worldwide basis to the BMJ Publishing Group Ltd to permit this article (if accepted) to be published in JECH and any other BMJPGL products and sublicences such use and exploit all subsidiary rights, as set out in our licence (http://group.bmj.com/products/journals/instructions-for-authors/licence-forms). 


\section{References}

1. Veronesi G, Ferrario MM, Kuulasmaa K, et al. Educational class inequalities in the incidence of coronary heart disease in Europe. Heart 2016;102:958-965.

2. Ferrario MM, Veronesi G, Kee F, et al. Determinants of social inequalities in stroke incidence across Europe: a collaborative analysis of 126635 individuals from 48 cohort studies. J Epidemiol Community Health 2017;71(12):1210-1216

3. Carter AR, Gill D, Davies NM, et al. Understanding the consequences of education inequality on cardiovascular disease: mendelian randomisation study. BMJ. 2019;365:11855.

4. Diderichsen F, Hallqvist J, Whitehead M. Differential vulnerability and susceptibility: how to make use of recent development in our understanding of mediation and interaction to tackle health inequalities. Int J Epidemiol. 2019;48:268-274.

5. Veronesi G, Tunstall-Pedoe H, Ferrario MM, et al. Combined effect of educational status and cardiovascular risk factors on the incidence of coronary heart disease and stroke in European cohorts: Implications for prevention. Eur J Prev Cardiol 2017;24:437-445.

6. Nordahl H, Osler M, Frederiksen BL, et al. Combined effects of socioeconomic position, smoking, and hypertension on risk of ischemic and hemorrhagic stroke. Stroke.

2014;45:2582-2587.

7. Nordahl H, Lange T, Osler M, et al. Education and Cause-specific Mortality. Epidemiology 2014;25:389-396.

8. Hussein M, Diez Roux A V, Mujahid MS, et al. Unequal Exposure or Unequal Vulnerability? Contributions of Neighborhood Conditions and Cardiovascular Risk Factors to Socioeconomic Inequality in Incident Cardiovascular Disease in the Multi-Ethnic Study of Atherosclerosis. Am J Epidemiol. 2018;187:1424-1437.

9. Schwartz J, Bellinger D, Glass T. Exploring potential sources of differential vulnerability and susceptibility in risk from environmental hazards to expand the scope of risk assessment. Am J Public Health. 2011;101 Suppl 1:S94-101.

10. McEwen BS, Stellar E. Stress and the individual. Mechanisms leading to disease. Arch Intern Med. 1993;153:2093-2101.

11. Juster RP, McEwen BS, Lupine SJ. Allostatic load biomarkers of chronic stress and impact in health and cognition. Neurosci Biobehav Rev. 2010;35(1):2-16.

12. Johnson SC, Cavallaro FL, Leon DA. A systematic review of allostatic load in relation to socioeconomic position: Poor fidelity and major inconsistencies in biomarkers employed. Soc Sci Med. 2017;192:66-73

13. Robertson T, Benzeval M, Whitley E, Popham F. The role of material, psychosocial and behavioral factors in mediating the association between socioeconomic position and allostatic load (measured by cardiovascular, metabolic and inflammatory markers). Brain Behav Immun. 2015;45:41-9. 
14. Jones HE, Schooling CM. Let's Require the "T-Word". American Journal of Public Health 2018;108:624

15. Zeller T, Hughes M, Tuovinen T, et al. BiomarCaRE: rationale and design of the European BiomarCaRE project including 300,000 participants from 13 European countries. Eur J Epidemiol 2014;29:777-790.

16. Evans A, Salomaa V, Kulathinal S, et al. MORGAM (an international pooling of cardiovascular cohorts). Int J Epidemiol 2005;34(1):21-27.

17. Veronesi G, Ferrario MM, Chambless LE, et al. for the MORGAM Project. Description and quality of baseline data: marital status, education and occupation. Available at: https://www.thl.fi/publications/morgam/qa/baseline/se_data/seqa.pdf

18. Karvanen J, Veronesi G, Kuulasmaa K. Defining thirds of schooling years in population studies. Eur J Epidemiol 2007;22:487-492.

19. Howard JT, Sparks PJ. Does allostatic load calculation method matter? Evaluation of different methods and individual biomarkers functioning by race/ethnicity and educational level. Am J Hum Biol 2016;28:627-635.

20. Corrao G, Bagnardi V, Zambon A, La Vecchia C. A meta-analysis of alcohol consumption and the risk of 15 diseases. Prev Med. 2004;38:613-619.

21. Sen B. Using the Oaxaca-Blinder Decomposition as an empirical tool to analyze racial disparities in obesity. Obesity 2014;22:1750-1755

22. Jann B. The Blinder-Oaxaca decomposition for linear regression models. The Stata Journal 2008;8:453-479

23. Ribeiro AI, Fraga S, Kelly-Irving M, et al. Neighbourhood socioeconomic deprivation and allostatic load: a multi-cohort study. Sci Rep. 2019;9(1):8790

24. Petrovic D, Pivin E, Ponte B, et al. Sociodemographic, behavioral and genetic determinants of allostatic load in a Swiss population-based study.

Psychoneuroendocrinology. 2016;67:76-85.

25. Lipowicz A, Szklarska A, Malina RM. Allostatic load and socioeconomic status in Polish adult men. J Biosoc Sci. 2014;46(2):155-67.

26. Hansen ÅM, Lund R, Bruunsgaard H, et al. Social gradient in allostatic load among Danish men and women in late midlife. J Aging Health. 2014;26(1):72-87.

27. Robertson T, Watts E. The importance of age, sex and place in understanding socioeconomic inequalities in allostatic load: Evidence from the Scottish Health Survey (2008-2011). BMC Public Health. 2016;16:126.

28. Seeman T, Merkin SS, Crimmins E, Koretz B, Charette S, Karlamangla A. Education, income and ethnic differences in cumulative biological risk profiles in a national sample of US adults: NHANES III (1988-1994). Soc Sci Med 2008;66(1):72-87. 
29. Suvarna B, Suvarna A, Phillips R, Juster RP, McDermott B, Sarnyai Z. Health risk behaviours and allostatic load: A systematic review. Neurosci Biobehav Rev. 2020;108:694711.

30. Maartje M. Schaap, Anton E. Kunst, et al. Female ever-smoking, education, emancipation and economic development in 19 European countries. Soc Sci Med 2009;68(7):1271-1278

31. Paluzie G, Sans S, Balañá L et al. Secular trends in smoking according to educational level between 1986 and 1996: The MONICA study. Catalonia (Spain). Gaceta Sanitaria 2001;15(4):303-311

32. Fiorito G, Polidoro S, Dugué PA, et al. Social adversity and epigenetic aging: a multicohort study on socioeconomic differences in peripheral blood DNA methylation. Sci Rep. 2017;7(1):16266.

33. Ding X, Barban N, Mills MC. Educational attainment and allostatic load in later life: Evidence using genetic markers. Prev Med. 2019;129:105866.

34. Gruenewald TL, Karlamangla AS, Hu P, et al. History of socioeconomic disadvantage and allostatic load in later life. Soc Sci Med. 2012;74(1):75-83.

35. Juster RP, Moskowitz DS, Lavoie J, D'Antono B. Sex-specific interaction effects of age, occupational status, and workplace stress on psychiatric symptoms and allostatic load among healthy Montreal workers. Stress. 2013;16(6):616-29.

36. Ferrario MM, Landsbergis P, Tsutsumi A, et al.; ICOH Scientific Committee on Cardiology in Occupational Health. Work environment: An opportunity for ground-breaking collaborations in cardiovascular disease prevention. Eur J Prev Cardiol. 2017;24(2_suppl):46

37. Hawkley LC, Lavelle LA, Berntson GG, Cacioppo JT. Mediators of the relationship between socioeconomic status and allostatic load in the Chicago Health, Aging, and Social Relations Study (CHASRS). Psychophysiology 2011;48(8):1134-1145.

38. Christensen AI, Ekholm O, Gray L, Glümer C, Juel K. What is wrong with nonrespondents? Alcohol-, drug- and smoking-related mortality and morbidity in a 12-year follow-up study of respondents and non-respondents in the Danish Health and Morbidity Survey. Addiction. 2015;110(9):1505-12.

39. Doan SN, Dich N, Evans GW. Childhood cumulative risk and later allostatic load: mediating role of substance use. Health Psychol. 2014;33(11):1402-1409.

doi:10.1037/a0034790 
Tables and Figures

Table 1. Distribution of allostatic load score, age, smoking, alcohol intake and prevalence of comorbidities across the educational classes. Men (left) and women (right), 35-74 years old.

\begin{tabular}{|c|c|c|c|c|c|c|c|c|}
\hline & \multicolumn{4}{|c|}{ Men, educational class } & \multicolumn{4}{|c|}{ Women, educational class } \\
\hline & Low & Intermediate & High & $\mathbf{p}$ & Low & Intermediate & High & $\mathbf{p}$ \\
\hline No. of subjects & 10131 & 6742 & 10146 & - & 10219 & 6733 & 9786 & - \\
\hline AL score, mean \pm SD & $0.26 \pm 4.1$ & $0.20 \pm 4.1$ & $-0.42 \pm 4.1$ & $<.0001$ & $0.66 \pm 4.4$ & $0.19 \pm 4.4$ & $-0.86 \pm 4.4$ & $<.0001$ \\
\hline \multicolumn{9}{|l|}{ Age class, \% } \\
\hline $35-44$ years & 24.2 & 18.2 & 24.8 & \multirow{3}{*}{$<.0001$} & 25.5 & 23.6 & 29.1 & \multirow{3}{*}{$<.0001$} \\
\hline $45-54$ years & 30.7 & 37.9 & 35.1 & & 35.2 & 32.9 & 33.7 & \\
\hline 55-74 years & 45.1 & 43.8 & 40.1 & & 39.4 & 43.1 & 37.3 & \\
\hline \multicolumn{9}{|l|}{ Smoking status, $\% \wedge$} \\
\hline Never smokers & 31.7 & 33.1 & 40.7 & \multirow{5}{*}{$<.0001$} & 59.3 & 62.0 & 59.4 & \multirow{5}{*}{$<.0001$} \\
\hline Former smokers & 35.3 & 37.8 & 37.9 & & 14.4 & 17.8 & 22.1 & \\
\hline 1-10 cigarettes/day & 7.5 & 7.4 & 6.3 & & 9.8 & 9.5 & 9.6 & \\
\hline 11-20 cigarettes/day & 17.4 & 14.6 & 10.7 & & 13.8 & 9.2 & 7.9 & \\
\hline$>20$ cigarettes/day & 8.2 & 7.1 & 4.4 & & 2.7 & 1.4 & 1.0 & \\
\hline \multicolumn{9}{|l|}{ Alcohol intake, drinks/day $\% \wedge$} \\
\hline 0 (Teetotallers) & 20.7 & 19.3 & 17.9 & \multirow{4}{*}{$<.0001$} & 49.6 & 44.0 & 39.3 & \multirow{4}{*}{$<.0001$} \\
\hline 1-2 drinks/day & 43.3 & 46.3 & 52.8 & & 45.7 & 50.9 & 55.2 & \\
\hline 3-4 drinks/day & 29.5 & 29.3 & 26.7 & & 4.6 & 5.0 & 5.4 & \\
\hline 5 or more drinks/day & 6.5 & 5.2 & 2.6 & & 0.1 & 0.1 & 0.1 & \\
\hline Presence of comorbidites, $\% \wedge$ & 60.5 & 59.2 & 56.1 & $<.0001$ & 57.3 & 54.7 & 46.6 & $<.0001$ \\
\hline Obese & 23.1 & 21.7 & 18.1 & $<.0001$ & 29.2 & 25.9 & 19.5 & $<.0001$ \\
\hline Elevated blood pressure* & 50.3 & 49.3 & 48.7 & 0.10 & 45.6 & 44.6 & 38.3 & $<.0001$ \\
\hline Positive history of diabetes & 5.3 & 4.8 & 4.3 & 0.005 & 4.3 & 3.8 & 3.1 & 0.0002 \\
\hline Positive history of CVD & 6.6 & 6.4 & 5.0 & $<.0001$ & 3.1 & 2.0 & 1.4 & $<.0001$ \\
\hline
\end{tabular}

$\wedge$ : age-adjusted proportion, estimated at the sample mean age of 52. $\mathrm{p}$ : $\mathrm{p}$-value testing the difference in the mean value of risk factors across the three educational classes. SD $=$ Standard deviation. ${ }^{\circ}: \mathrm{BMI}>30 \mathrm{~kg} / \mathrm{m} 2{ }^{*}$ *: elevated $\mathrm{BP}(\mathrm{SBP}>140$ or DBP $>90)$ or taking antihypertensive drugs. 
Table 2a: Details of the Oaxaca-Blinder Decomposition of Allostatic Load score difference between low and high education, for smoking and alcohol intake. Men, 35-74 years old at baseline, in the low $(n=10131)$ and in the high educational class $(n=10146)$

\begin{tabular}{|c|c|c|c|c|c|c|c|c|}
\hline \multirow[b]{3}{*}{ Smoking } & \multicolumn{4}{|c|}{ Differential Exposure } & \multicolumn{4}{|c|}{ Differential Susceptibility } \\
\hline & \multirow{2}{*}{$\begin{array}{c}\text { Absolute }^{1} \\
0.036\end{array}$} & \multicolumn{2}{|c|}{$95 \% \mathrm{CI}$} & \multirow{2}{*}{$\begin{array}{c}\begin{array}{c}\text { Relative}^{\wedge} \\
\mathbf{( \% )}\end{array} \\
5.2\end{array}$} & \multirow{2}{*}{$\begin{array}{c}\text { Absolute }^{2} \\
0.177\end{array}$} & \multicolumn{2}{|c|}{$95 \% \mathrm{CI}$} & \multirow{2}{*}{$\begin{array}{c}\text { Relative }^{\wedge} \\
\mathbf{( \% )}\end{array}$} \\
\hline & & 0.013 & 0.058 & & & 0.069 & 0.286 & \\
\hline Never smokers & 0.032 & 0.018 & 0.047 & 4.7 & 0.147 & 0.063 & 0.230 & 21.5 \\
\hline Former smokers & -0.004 & -0.008 & 0.000 & -0.6 & 0.065 & -0.013 & 0.144 & 9.6 \\
\hline $1-10$ cigs/day & -0.003 & -0.006 & 0.000 & -0.4 & 0.018 & -0.004 & 0.040 & 2.7 \\
\hline 11-20 cigs/day & -0.007 & -0.018 & 0.004 & -1.0 & -0.025 & -0.055 & 0.005 & -3.7 \\
\hline$>20$ cigs $/$ day & 0.017 & 0.008 & 0.026 & 2.5 & -0.028 & -0.046 & -0.010 & -4.1 \\
\hline Alcohol intake & 0.013 & -0.006 & 0.031 & 1.9 & 0.109 & -0.029 & 0.247 & 15.9 \\
\hline 0 (Teetotallers) & -0.001 & -0.006 & 0.004 & -0.1 & 0.020 & -0.024 & 0.065 & 3.0 \\
\hline 1-2 drinks/day & 0.008 & -0.005 & 0.021 & 1.1 & 0.094 & -0.016 & 0.204 & 13.8 \\
\hline 3-4 drinks/day & -0.005 & -0.009 & 0.000 & -0.7 & 0.002 & -0.058 & 0.063 & 0.3 \\
\hline 5 or more & 0.011 & 0.001 & 0.020 & 1.6 & -0.008 & -0.019 & 0.004 & -1.1 \\
\hline Aggregate contribution* & 0.048 & 0.020 & 0.077 & 7.1 & 0.286 & 0.119 & 0.453 & 41.9 \\
\hline
\end{tabular}

$\wedge^{\wedge}$ : Ratio between the absolute coefficient and the un-adjusted mean difference in AL score between low and high education [0.68; 95\%CI: 0.57; 0.79].

*: The difference in educational class intercepts accounts for a residual 45\%. Age and center account for 5.8\%. All totaling $100 \%$

1: a positive (negative) coefficient is the expected reduction (increase) in social gradient in AL score if low educated if low educated had, on average, the same prevalence of risk factors as high educated men.

2: a positive (negative) coefficient is the expected reduction (increase) in social gradient in AL score if the effect of risk factor on AL in low educated men was the same as in high educated men

In bold: results supporting statistical significance. 
Table 2b: Details of the Oaxaca-Blinder Decomposition of Allostatic Load score difference between low and high education, for smoking and alcohol intake. Women, 35-74 years old at baseline, in the low $(n=10219)$ and in the high educational class $(n=9786)$

\begin{tabular}{|c|c|c|c|c|c|c|c|}
\hline \multirow[b]{3}{*}{ Smoking } & \multicolumn{4}{|c|}{ Differential Exposure } & \multicolumn{3}{|c|}{ Differential Susceptibility } \\
\hline & Absolute $^{1}$ & $95 \%$ & $\% \mathrm{CI}$ & $\begin{array}{c}\text { Relative }^{\wedge} \\
(\%)\end{array}$ & Absolute $^{2}$ & $95 \% \mathrm{CI}$ & $\begin{array}{c}\text { Relative }^{\wedge} \\
(\%)\end{array}$ \\
\hline & 0.026 & 0.003 & 0.049 & 1.7 & 0.095 & $-0.093 \quad 0.282$ & 6.2 \\
\hline Never smokers & 0.000 & -0.001 & 0.000 & 0.0 & 0.103 & $-0.043 \quad 0.248$ & 6.8 \\
\hline Former smokers & 0.009 & -0.006 & 0.025 & 0.6 & 0.026 & $\begin{array}{ll}-0.039 & 0.091\end{array}$ & 1.7 \\
\hline $1-10$ cigs/day & 0.000 & -0.006 & 0.006 & 0.0 & -0.017 & $-0.052 \quad 0.017$ & -1.1 \\
\hline 11-20 cigs/day & 0.000 & -0.012 & 0.012 & 0.0 & -0.017 & $-0.046 \quad 0.011$ & -1.1 \\
\hline$>20$ cigs/day & 0.016 & 0.009 & 0.024 & 1.1 & 0.001 & $\begin{array}{ll}-0.007 & 0.009\end{array}$ & 0.1 \\
\hline Alcohol intake & 0.066 & 0.048 & 0.085 & 4.4 & -0.051 & -0.1940 .092 & -3.3 \\
\hline 0 (Teetotallers) & 0.037 & 0.021 & 0.053 & 2.4 & -0.017 & $-0.098 \quad 0.063$ & -1.2 \\
\hline 1-2 drinks/day & 0.029 & 0.014 & 0.044 & 1.9 & -0.040 & $-0.154 \quad 0.074$ & -2.6 \\
\hline 3 or more drinks/day & 0.000 & -0.002 & 0.003 & 0.0 & 0.006 & -0.0120 .025 & 0.4 \\
\hline Aggregate contributon* & 0.092 & 0.063 & 0.122 & 6.1 & 0.044 & $\begin{array}{ll}-0.185 & 0.272 \\
\end{array}$ & 2.9 \\
\hline
\end{tabular}

$\wedge$ : Ratio between the absolute coefficient and the un-adjusted mean difference in AL score between low and high education [1.52; 95\%CI: 1.40; 1.64$]$.

*: The difference in educational class intercepts accounts for a residual $79 \%$. Age and center account for $11.8 \%$. All totaling $100 \%$

1: a positive (negative) coefficient is the expected reduction (increase) in social gradient in AL score if low educated had, on average, the same prevalence of risk factors as high educated women.

2: a positive (negative) coefficient is the expected reduction (increase) in social gradient in AL score if the effect of risk factor on AL in low educated men was the same as in high educated women

In bold: results supporting statistical significance. 
Table 3: Aggregate contribution of smoking and alcohol intake to the Oaxaca-Blinder Decomposition of Allostatic Load difference between low and high education. System-specific sub-scores and total Allostatic Load score.

Men (above) and women (below), 35-74 years old at baseline

\begin{tabular}{|c|c|c|c|c|c|c|c|c|}
\hline & \multirow[b]{2}{*}{$\Delta \mathrm{ED}(95 \% \mathrm{CI})$} & \multicolumn{4}{|c|}{ Differential Exposure } & \multicolumn{3}{|c|}{ Differential Susceptibility } \\
\hline & & Absolute $^{1}$ & $95 \%$ & & $\begin{array}{c}\text { Relative }^{\wedge} \\
\text { (\%) }\end{array}$ & Absolute $^{2}$ & $95 \% \mathrm{CI}$ & $\begin{array}{c}\text { Relative }^{\wedge} \\
\text { (\%) }\end{array}$ \\
\hline \multicolumn{9}{|l|}{ Men } \\
\hline Cardiovascular system & $0.13(0.08 ; 0.18)$ & 0.00 & -0.01 & 0.02 & 2.1 & 0.01 & -0.070 .08 & 6.0 \\
\hline Metabolic system & $0.37(0.29 ; 0.44)$ & -0.01 & -0.03 & 0.01 & -1.8 & 0.25 & $\begin{array}{ll}0.14 & 0.37\end{array}$ & 69.3 \\
\hline Inflammation & $0.19(0.16 ; 0.22)$ & 0.05 & 0.04 & 0.06 & 27.7 & 0.02 & -0.020 .06 & 12.9 \\
\hline Total AL score & $0.68(0.57 ; 0.79)$ & 0.05 & 0.02 & 0.08 & 7.1 & 0.29 & 0.120 .45 & 41.9 \\
\hline \multicolumn{9}{|l|}{ Women } \\
\hline Cardiovascular system & $0.32(0.27 ; 0.37)$ & -0.01 & -0.02 & 0.00 & -3.3 & -0.04 & -0.140 .06 & -12.0 \\
\hline Metabolic system & $0.98(0.90 ; 1.06)$ & 0.08 & 0.06 & 0.10 & 8.4 & 0.09 & $-0.07 \quad 0.24$ & 8.8 \\
\hline Inflammation & $0.23(0.20 ; 0.25)$ & 0.02 & 0.01 & 0.03 & 9.4 & 0.00 & $-0.06 \quad 0.05$ & -1.9 \\
\hline Total AL score & $1.52(1.40 ; 1.64)$ & 0.09 & 0.06 & 0.12 & 6.1 & 0.04 & $\begin{array}{ll}-0.18 & 0.27\end{array}$ & 2.9 \\
\hline
\end{tabular}

1: a positive (negative) coefficient is the expected reduction (increase) in social gradient in AL score if low educated on average, the same prevalence of risk factors as high educated individuals.

2: a positive (negative) coefficient is the expected reduction (increase) in social gradient in AL score if the effect of risk factor on AL in low educated individuals was the same as in high educated individuals.

Cardiovascular system: systolic and diastolic BP

Metabolic system: Body Mass Index, Total cholesterol, HDL-cholesterol, triglycerides, glucose.

Inflammation: CRP.

$\triangle \mathrm{ED}=$ un-adjusted mean difference in Allostatic Load score between low and high education, with $95 \%$ confidence intervals

$\wedge$ : Ratio between the absolute coefficient and the un-adjusted mean difference in AL score between low and high education $(\Delta E D)$

In bold: results supporting statistical significance. 
Table 4: Aggregate contribution of smoking and alcohol intake to the Oaxaca-Blinder Decomposition of Allostatic Load score difference between low and high education, by age group and according to presence of comorbidities.

Men (above) and women (below), 35-74 years old at baseline

\begin{tabular}{|c|c|c|c|c|c|c|c|c|c|c|}
\hline & \multirow[b]{2}{*}{$\mathbf{N}$} & \multirow[b]{2}{*}{$\Delta \operatorname{ED}(95 \% \mathrm{CI})$} & \multicolumn{4}{|c|}{ Differential Exposure } & \multicolumn{4}{|c|}{ Differential Susceptibility } \\
\hline & & & Absolute $^{1}$ & \multicolumn{2}{|c|}{$95 \%$ CI } & \multirow[t]{2}{*}{$\begin{array}{c}\text { Relative } \\
(\%)\end{array}$} & Absolute $^{2}$ & \multicolumn{2}{|c|}{$95 \%$ CI } & $\begin{array}{c}\text { Relative }^{\wedge}(\%) \\
\end{array}$ \\
\hline Men & & & & & & & & & & \\
\hline \multicolumn{11}{|l|}{ Age group } \\
\hline 35-44 years old & 4973 & $0.93(0.71 ; 1.15)$ & 0.17 & 0.10 & 0.25 & 18.7 & 0.17 & -0.15 & 0.50 & 18.5 \\
\hline $45-54$ years old & 6673 & $0.58(0.39 ; 0.78)$ & 0.08 & 0.01 & 0.14 & 13.0 & 0.45 & 0.17 & 0.73 & 76.9 \\
\hline 55-74 years old & 8631 & $0.52(0.36 ; 0.69)$ & -0.02 & -0.06 & 0.01 & -4.5 & 0.32 & 0.03 & 0.61 & 61.0 \\
\hline \multicolumn{11}{|l|}{ Comorbidities* } \\
\hline None & 8535 & 0.53 (0.39; 0.67) & 0.09 & 0.04 & 0.13 & 16.7 & 0.22 & 0.00 & 0.44 & 41.2 \\
\hline At least one & 11742 & $0.41(0.28 ; 0.54)$ & 0.02 & -0.01 & 0.05 & 5.5 & 0.23 & 0.03 & 0.42 & 54.9 \\
\hline \multicolumn{11}{|l|}{ Women } \\
\hline \multicolumn{11}{|l|}{ Age group } \\
\hline 35-44 years old & 5448 & 1.52 (1.31; 1.73) & 0.10 & 0.03 & 0.16 & 6.3 & 0.17 & -0.25 & 0.58 & 10.9 \\
\hline $45-54$ years old & 6886 & $1.41(1.21 ; 1.61)$ & 0.14 & 0.08 & 0.19 & 9.6 & -0.10 & -0.46 & 0.27 & -6.8 \\
\hline 55-74 years old & 7671 & $1.27(1.10 ; 1.45)$ & 0.07 & 0.02 & 0.11 & 5.1 & 0.09 & -0.34 & 0.53 & 7.4 \\
\hline \multicolumn{11}{|l|}{ Comorbidites* } \\
\hline None & 9687 & $1.11(0.98 ; 1.24)$ & 0.10 & 0.06 & 0.13 & 8.8 & 0.02 & -0.22 & 0.26 & 1.9 \\
\hline At least one & 10318 & $0.82(0.68 ; 0.96)$ & 0.08 & 0.04 & 0.11 & 9.5 & 0.05 & -0.26 & 0.36 & 6.1 \\
\hline
\end{tabular}

1: a positive (negative) coefficient is the expected reduction (increase) in social gradient in AL score if low educated had on average, the same prevalence of risk factors as high educated individuals.

2: a positive (negative) coefficient is the expected reduction (increase) in social gradient in AL score if the effect of risk factor on AL in low educated individuals was the same as in high educated individuals.

$\Delta \mathrm{ED}=$ un-adjusted mean difference in Allostatic Load score between low and high education

$\wedge$ : Ratio between the absolute coefficient and the un-adjusted mean difference in AL score between low and high education $(\Delta \mathrm{ED})$

In bold: results supporting statistical significance.

*: any of the following: obesity, elevated blood pressure, diabetes, cardiovascular disease (see methods). Detailed results by condition are in the Supplementary Material (Table S6) 